\title{
Role of Amino Acid Metabolism in the Virulence of Human Pathogenic Fungi
}

\author{
Enrico Garbe $^{1,2} \cdot$ Slavena Vylkova ${ }^{1,2}$ \\ Published online: 17 July 2019 \\ (C) The Author(s) 2019, corrected publication 2019
}

\begin{abstract}
Purpose of the Review The success of Candida albicans, Aspergillus fumigatus, and Cryptococcus neoformans as fungal pathogens depends on their rapid adaptation to host microenvironments, through metabolic remodeling, stress resistance, and expression of virulence determinants. Amino acids represent an abundant nitrogen and carbon source within the host; however, their acquisition by fungi is a very complex process that interconnects several sensory and uptake systems and downstream pathways. In this review, we will summarize the current knowledge concerning this topic, identify gaps or discrepancies, and discuss future research directions.

Recent Findings Aside from supporting basic cellular functions, the utilization of many amino acids has a direct effect on fungal pathogenicity by triggering key virulence traits, including hyphal morphogenesis and biofilm growth in $C$. albicans, capsule formation in C. neoformans, and melanization in A. fumigatus.

Summary Although many components of amino acid sensing and metabolism are fungal specific, their importance in infection and potential as candidates for antifungal drug development require further investigation.
\end{abstract}

Keywords Amino acid metabolism $\cdot$ Candida $\cdot$ Cryptococcus $\cdot$ Aspergillus $\cdot$ Virulence $\cdot$ Nutrient sensing

\section{Introduction}

Invasive fungal infections are an increasing human health problem, particularly among individuals with impaired immunity. The main agents causing these infections are the ascomycetes, Candida albicans $(\mathrm{Ca})$ and Aspergillus fumigatus (Af), and the basidiomycete, Cryptococcus neoformans $(\mathrm{Cn})$, which account for more than 1.5 million invasive infections per year with mortality rates between 20 and 95\% [1]. Poor diagnosis, intrinsic or acquired antifungal resistance, and the limited number of available antifungal drugs are the main challenges in their clinical treatment.

This article is part of the Topical Collection on Mycology

Slavena Vylkova

slavena.vylkova@leibniz-hki.de

1 NWG Host Fungal Interfaces, Friedrich Schiller University, Jena, Germany

2 Septomics Research Centre, Leibniz Institute for Natural Product Research and Infection Biology - Hans Knoell Institute, Jena, Germany
Besides the primary virulence factors, which have been studied more extensively, metabolic versatility represents an integral part of fungal pathogenicity, since it provides the platform for nutrient assimilation and adaptation to host-imposed stress and antifungal drugs [2-13]. For the pathogenic fungi reviewed here, metabolic adaptations have evolved dependent on their environmental reservoir, generating the basis for the differences in nutrient utilization. C. albicans is a commensal of humans where it colonizes several distinct host niches and has developed a remarkable metabolic plasticity that governs its survival within the host. In contrast to this tight association with humans, C. neoformans and A. fumigatus are ubiquitous environmental fungi that are commonly found in soil, compost, and bird guano, which enter the human via the accidental inhalation of spores. Despite the differences in their primary natural habitats, all three fungi have developed multiple mechanisms for fast and effective acquisition of amino acids, available in free form or as part of host proteins in every host niche [14-18]. Aside from supporting growth, amino acid metabolism can trigger virulence traits, including hyphal morphogenesis, biofilm formation, melanization, and capsule formation $[19,20 \bullet, 21 \bullet, 22 \bullet, 23 \bullet]$. Importantly, pathogenic fungi possess multiple amino acid transporters and biosynthetic pathways 
not present in humans and essential for fungal growth, pointing to their potential as drug targets [24]. In this review, we will highlight and critically discuss the latest findings in sensing, uptake and metabolism of amino acids, and their role in fungal virulence, and suggest future research perspectives. Given that the topic is best investigated in C. albicans, we will discuss this organism primarily and include interesting findings for C. neoformans and A. fumigatus where available.

\section{Amino Acid Sensing and Regulation of Their Metabolism}

Fungi have evolved several sensing mechanisms and regulatory circuits to monitor and utilize extracellular amino acids, like the nitrogen catabolite repression (NCR), the target of rapamycin (TOR) pathway, and SPS and transceptormediated amino acid sensing (Table 1). They are all wellstudied in the model yeast Saccharomyces cerevisiae (Sc) and therefore used here as a reference point [36]. The role of NCR and TOR in pathogenic fungi was summarized recently and will be mentioned only briefly [37].
Many fungi preferentially utilize ammonium, glutamine, and glutamate as nitrogen sources, while nitrates, nitrites, purines, amides, other amino acids, and proteins are used when primary nitrogen sources are limited. The utilization of secondary nitrogen sources is directed by a global control mechanism named NCR and mediated by the GATA-type transcription factors ScGat1 and ScGln3, which positively regulate the expression of permeases and pathway-specific catabolic enzymes upon nitrogen starvation $[36,38,39]$. S. cerevisiae orthologs governing similar regulatory circuits are described for all three pathogenic fungi [27-30, 40, 41]. It has been demonstrated that $\mathrm{CaGln} 3$ is also required for nitrogen starvation-induced filamentation and full virulence in a murine model, while CnGat1 is dispensable for proline and arginine utilization and survival in the host [27-29, 40, 41]. The A. fumigatus ortholog AfareA is required for virulence in a murine model of pulmonary aspergillosis [30].

The TOR pathway represents the main sensor for the intracellular nutrient state and mediates cellular responses, including alterations in the nitrogen and amino acid metabolism. The integration of extra- and intracellular nutrient-derived signals into other signaling and regulatory pathways is mediated via the nutrient-sensitive Tor kinase ScTor1 [36, 42]. Although

Table 1 Orthologs of the S. cerevisiae main regulatory systems for amino acid metabolism in C. albicans, C. neoformans, and A. fumigatus and their relevance for host-pathogen interaction

\begin{tabular}{|c|c|c|c|c|c|c|}
\hline \multirow[t]{2}{*}{ S. cerevisiae } & \multicolumn{2}{|l|}{ C. albicans } & \multicolumn{2}{|c|}{ C. neoformans } & \multicolumn{2}{|c|}{ A. fumigatus } \\
\hline & Orthologs & Phenotype & Orthologs & Phenotype & Orthologs & Phenotype \\
\hline SPS system & $\begin{array}{l}\text { CaSsy1 } \\
\text { CaSsy5 } \\
\text { CaPtr3 } \\
\text { CaStp1 } \\
\text { CaStp2 }\end{array}$ & $\begin{array}{l}\text { stp } 2 \triangle \text { is attenuated in murine } \\
\text { intravenous infection model [25]; } \\
\text { stp } 2 \triangle \text { and ssy } 1 \triangle \text { are impaired in } \\
\text { escape from macrophages }[25,26] \\
\text { ssyl } \triangle, \text { ssy } 5 \triangle \text {, and ptr } 3 \triangle \text { are } \\
\text { slightly attenuated in murine } \\
\text { intravenous infection model }[26]\end{array}$ & - & - & - & - \\
\hline NCR & $\begin{array}{l}\text { CaGat1 } \\
\text { CaGIn3 }\end{array}$ & $\begin{array}{l}\text { Highly attenuated in murine } \\
\text { intravenous infection model }[27,28]\end{array}$ & CnGat1 & $\begin{array}{l}\text { Wild type phenotype in murine } \\
\text { intranasal and in vitro macrophage } \\
\text { infection model [29] }\end{array}$ & AfAreA & $\begin{array}{l}\text { Attenuated in } \\
\text { intranasal } \\
\text { infection } \\
\text { model in } \\
\text { neutropenic } \\
\text { mice [30] }\end{array}$ \\
\hline TOR & CaTor1 & - & CnTor1 & $\begin{array}{l}\text { Wild type phenotype in systemic } \\
\text { murine and rabbit infection models } \\
\text { [46]; Impaired TOR pathway results } \\
\text { in attenuated virulence in murine } \\
\text { intravenous infection model [31] }\end{array}$ & AfTor & - \\
\hline GAAC & CaGen 4 & $\begin{array}{l}\text { Attenuated in murine intravenous } \\
\text { infection model [32] }\end{array}$ & - & - & AfCpcA & $\begin{array}{l}\text { Attenuated in } \\
\text { murine } \\
\text { pulmonary } \\
\text { infection } \\
\text { model [33] }\end{array}$ \\
\hline ScGap1 & CaGap1-6 & - & CnAap1-8 & $\begin{array}{l}\text { oap } 4 \Delta / \text { oap } 5 \Delta \text {, oap } 1 \Delta / \text { aap } 2 \Delta \text { and } \\
\text { oap } 8 \Delta \text { strains are attenuated in } \\
\text { G. melonella model, aap } 4 \Delta / \text { aap } 5 \Delta \\
\text { in murine inhalation model }[34,35]\end{array}$ & AfGap1 & - \\
\hline
\end{tabular}


CaTor1 has not been studied in detail, it is assumed that it likewise influences the expression of NCR-associated genes and amino acid permeases (AAPs) [43], while Aftor was shown to be involved in amino acid metabolism [44]. The nitrogen or amino acid metabolism-related functions of CnTor1 have not been defined. The TOR pathway also influences fungal virulence, as demonstrated by its involvement in nutrient-dependent regulation of adhesins, hyphal morphogenesis, and chlamydospore formation in C. albicans [43, 45]. TOR signaling is also required for fluconazole tolerance and full virulence in a murine model in C. neoformans [31, 46]. Closely connected to TOR signaling is the general amino acid control (GAAC), which in S. cerevisiae induces amino acid biosynthesis upon starvation in a process that is mediated by the transcription factor ScGen4 [36, 47]. Similarly, $\mathrm{CaGen} 4$ is required for filamentous growth in response to amino acid starvation and for full virulence in systemic model of candidiasis $[32,48]$. In A. fumigatus, cpcA encodes a functional ortholog of ScGen4 [33]. To our knowledge, a functional ortholog of ScGen4 is not described in C. neoformans and was also not identified via BLAST analysis performed within in the scope of this review (Table 1).

The Ssy1/Ptr3/Ssy5 (SPS) sensor system is the key element for monitoring extracellular amino acid concentrations in $S$. cerevisiae. Otherwise the organism is not really clear. [36, 49]. The system contains a functionally adapted, membrane-bound AAP named ScSsy1 which is activated upon increased extracellular concentrations of various amino acids, though the degree of activation varies by amino acid. SPS activation results in proteolytic cleavage of the inhibitory $\mathrm{N}$-terminal domains of the cytosol retained transcription factors ScStp1 and ScStp2, which have redundant activity. Subsequently, ScStp1 and ScStp 2 translocate into the nucleus and induce the expression of genes required for amino acid or protein utilization. The functionality of the system relies on the activity of ScShr3, an ER chaperone required for membrane localization of AAPs, including ScSsy1, which is therefore considered an upstream regulator of the SPS system [50, 51]. Like $S$. cerevisiae, C. albicans also senses extracellular amino acids via the SPS sensor system. Although CaSsy1 and ScSsyl share a high degree of homology in the C-terminal domains, their N-terminal-sensing domains are highly divergent [52]. It is likely that this explains the altered CaSsyl substrate specificity: the highest induction occurs in the presence of Arg, Asn, Asp, Gln, Glu, His, Lys, and Ser, while the other amino acids fail to stimulate the sensor [22, 52]. Further differences can be found in the downstream effectors CaStp1 and CaStp2 which, contrary to $S$. cerevisiae, exhibit a clear dichotomy in their regulatory activity: while the CaStp1 regulon contains genes required for protein utilization; like secreted aspartyl proteases (SAP) and oligopeptide transporters
(OPT), CaStp2 is required for the expression of AAPs [53]. It could be hypothesized that the divergence is the result of the adaptation of $C$. albicans to the human host and is also relevant for virulence. Indeed, multiple components of the $C$. albicans SPS-sensing pathway have been implicated in virulence-for example CaSsyl and CaStp2 are reported to be essential for macrophage phagosome alkalinization and immune cell evasion [25•, 26]. Additionally, deletion of $\mathrm{CaCSH} 3$, the ortholog of ScSHR3, resulted in impaired utilization of tryptophan and proline as nitrogen sources, filamentation, and reduced virulence in a murine model [54]. Ramachandra and colleagues have found several novel connections between $C$. albicans genes involved in the SPS-dependent amino acid sensing and NCR-mediated regulation, but the importance of such network interference and its impact on pathogenicity requires further characterization $[28,55]$.

To date, no functional SPS sensor systems have been described in C. neoformans or A. fumigatus nor could orthologs be found in their genomes via BLAST analysis conducted within the scope of this review, although it has been reported that C. neoformans possesses orthologs for ScSsy1 and ScStp2 [56•]. Putative orthologs of ScSHR3 are present in both C. neoformans and A. fumigatus, but have not been characterized. Therefore, it remains unclear how extracellular amino acids and oligopeptides are detected in these two organisms.

Another sensing mechanism for extracellular amino acids is provided by transceptors, which act simultaneously as transporters and receptors. ScGap1 is described as a broadspecificity transceptor for all L-amino acids, some D-amino acids, citrulline, and even some polyamines [57, 58]. ScGapl undergoes a complex activation cycle involving internalization, ubiquitination, retention at vacuolar membranes, and subsequent re-recruitment to the cell membrane based on nitrogen source availability, and is tightly regulated by $\operatorname{NCR}[36,59]$. Additionally, ScGap1 acts as an inducer of the cAMP/PKA pathway in response to amino acid sensing $[60,61]$. However, the precise mechanism by which ScGap1 activates downstream signaling pathways remains elusive [61, 62].

Initially, $\mathrm{CaGAP1}$ was considered to be the primary, functional ortholog of ScGAP1 and linked to GlcNAc-induced hyphal morphogenesis via the cAMP/PKA pathway [63]. However, a recent study revealed six ScGap1 orthologs in C. albicans, with CaGap1, CaGap2, and CaGap6 also possessing transceptor activity, as demonstrated by their activation of PKA in complementation experiments performed in $S$. cerevisiae [64]. Interestingly, proof of cAMP/PKA induction via these orthologs in $C$. albicans could be not provided so far [65•]. Currently, it is unknown if $C$. neoformans and A. fumigatus have functional transceptors. A. fumigatus encodes for at least one uncharacterized ScGAP1 ortholog, while C. neoformans possesses a variety of amino acid permeases, but none seem to act as a transceptor $[34,56 \bullet]$. 


\section{Utilization and Uptake of Amino Acids and Oligopeptides in the Context of Pathogenesis}

Fungi possess multiple secreted proteinases involved in nutrient utilization from host tissues. Secreted aspartic proteinases, in particular, foster the extracellular cleavage of proteins to oligopeptides and single amino acids, facilitating their uptake by fungi [66]. The SAP family in C. albicans encodes for 10 proteases and is well-studied regarding its impact on pathogenicity and fungal growth [67]. The hypothesis that SAPs liberate nutrients is supported by the fact that the eight C. albicans OPTs display differential expression in the presence of bovine serum albumin (BSA) [55, 68]. Indeed, mutant strains containing single knockouts for CaSAP1, CaSAP2, and CaSAP3 or a CaSAP4/5/6 triple knockout show impaired growth on BSA as the sole nitrogen source $[69,70]$. Similarly, a triple knockout of $\mathrm{CaOPT1/2/3}$ fails to grow on proteins as the sole nitrogen source [68].

Generally, proteinase activity also appears to be important during C. neoformans infection, since degradation of extracellular matrix and collagen has been observed in murine infection models and is viewed as a facilitator for establishing infections in the central nervous system via permeabilization of epithelial barriers [71-73]. Further, C. neoformans produces several secreted proteases involved in the utilization of immunoglobulin and complement proteins as the sole nitrogen and carbon source in vitro [74]. The aspartyl proteinase CnMay1 and the carboxypeptidase $\mathrm{CnCxd} 1$ have a broad specificity and are considered to supply $C$. neoformans with free amino acids liberated from extracellular protein sources and a strain lacking CnMAYl was attenuated in a competition infection assay [75]. The functionality of oligopeptide transporters in C. neoformans virulence has not been investigated, although the fungal genome encodes for six predicted OPTs [76]. However, CnOpt1 was reported to be involved in a quorum-sensing process, thereby regulating the expression of virulence-associated genes [77].

A. fumigatus is a saprophytic organism that secretes a plethora of extracellular proteinases to provide nutrients for the cell. Its genome encodes for approx. 111 predicted proteases, which are at least partially induced upon various protein substrates and NCR-regulated via AfPrtT [78-83]. A mutant lacking AfprtT displayed markedly reduced expression of six secreted proteinases (Afalp, Afmep, Afdpp 4, AfcpdS, AFUA 2G17330, and AFUA 7G06220) on protein substrates in vitro and reduced damage of lung epithelial cells and erythrocytes, but did not exhibit virulence defects in a murine model [81]. Generally, the role of proteinases in A. fumigatus virulence and nutrient acquisition is not well understood and difficult to investigate, due to their sheer number and functional redundancy. Nevertheless, the secretion of proteinases in vivo is shown to trigger host allergic responses $[82,84]$. Interestingly, a mutant strain lacking all eight predicted members of the $O P T$ gene family showed no growth defect on various nitrogen sources or virulence attenuation in a murine model of pulmonary aspergillosis [83]. This suggests either alternative uptake mechanisms or very efficient cleavage of host proteins to single amino acids.

In fungi, transport of amino acids into the cell occurs via AAPs with various efficiency and substrate specificity. The C. albicans genome contains at least 28 open reading frames (ORFs) encoding for AAPs [65•]. From this family, CaGap16 have been comprehensively studied $[64,65]$. CaGap2 was identified as the functional ortholog of the general amino acid permease ScGap1, since it promoted growth on all tested amino acids as the sole source of nitrogen in a $S$. cerevisiae complementation assay [64]. This function as a general amino acid permease was further verified in C. albicans, where a strain lacking $\mathrm{CaGAP} 2$ failed to grow on Phe, Met, Val, Leu, Tyr, Trp, and Lys as the sole nitrogen source [65•]. CaGap6 also displayed a broad specificity, while CaGap1 and CaGap4 utilized only specific amino acids [64]. Interestingly, CaGap3 and CaGap5 were dispensable for growth on amino acids as a nitrogen source, yet their expression was nitrogen sourcedependent, suggesting that they may complement the activity of the broad specificity permeases in as of yet unidentified host niches or conditions. The prominent role of CaGap2 is also reflected in its regulatory pattern: upon a shift from nitrogen starvation to nitrogen replete conditions, CaGap2 was downregulated at the transcript level and rapidly internalized, while the other $G A P$ s were upregulated and the corresponding proteins remained on the plasma membrane (except for CaGap5). Interestingly, if this shift was reversed, only $\mathrm{CaGAP2}$ and $\mathrm{CaGAP6}$ were significantly induced [65 ${ }^{\circ}$. Moreover, the expression of all six CaGAPs appears to be dependent on SPS activity in contrast to $\mathrm{Sc} G A P 1[85,86]$ (own unpublished data). Furthermore, at least CaGap2 is directly regulated by $\mathrm{CaGat} 1$ and $\mathrm{CaGln} 3$ [28].

Although various AAPs and transporters exist in C. albicans besides the GAP family, they remain poorly characterized. Based on predictions from S. cerevisiae, the $C$. albicans non-GAP permeases are considered to possess higher substrate specificity and possibly facilitate a rapid and precise adaptation to changing host environments in order to overcome the nutritional immunity induced by the host. An interesting example is CaPUT4, an ortholog of the $S$. cerevisiae high-affinity proline permease gene ScPUT4: although this permease is unstudied, it was shown that proline can serve simultaneously as the sole carbon and nitrogen source and its utilization is independent of NCR regulation [87•]. Further, proline sensing and uptake was independent of the SPS sensor system and the growth on proline as the sole nitrogen source was only slightly affected in a CaCSH3 knockout [53, 54]. 
The importance of proline in C. albicans pathogenesis was recently further underscored by the finding that proline catabolism-mediated cAMP/PKA activation induces hyphal morphogenesis [22•]. This study presents a new mechanism of amino acid-directed hyphal morphogenesis, where mitochondrial proline catabolism feeds the TCA cycle and leads to an accumulation of electron donors, which are subsequently used for the generation of ATP. The accumulated ATP then stimulates hyphal morphogenesis via the PKA pathway
(Fig. 1) [22•]. It is further hypothesized that arginine uptake induces filamentation via the same pathway, since arginine is intracellularly converted to proline. Mitochondrial catabolism in some ways resembles a glucose-dependent process where high levels of glucose $(2 \%)$ inhibit mitochondrial catabolism, while low concentrations $(0.2 \%)$ activate oxidative respiration and stimulate proline- and arginine-induced filamentation [22•]. Nevertheless, filamentation is undoubtedly visible even in the presence of higher glucose concentrations, where

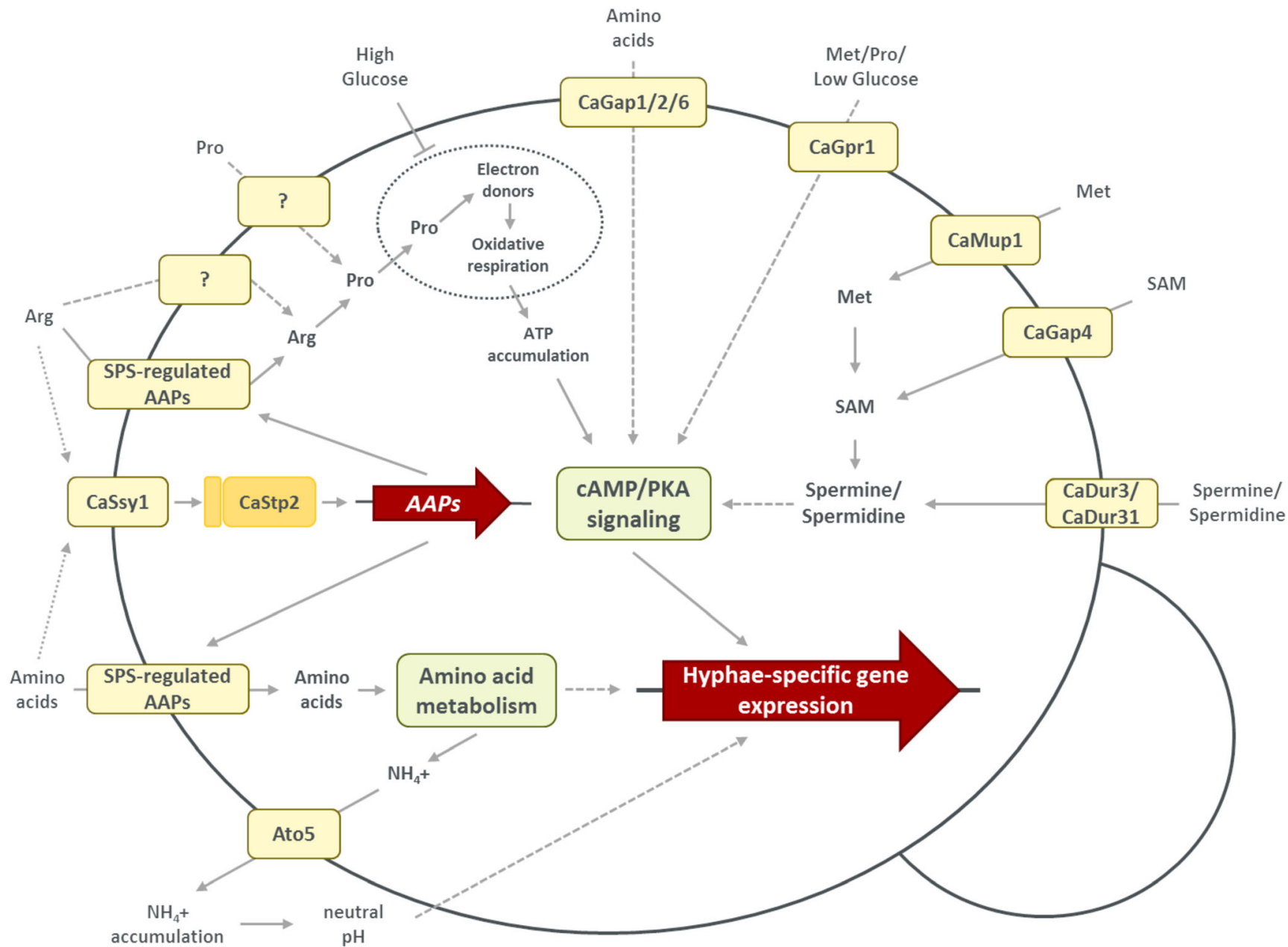

Fig. 1 Amino acid-induced hyphal morphogenesis in C. albicans. Given here is an overview of the described pathways for amino acidinduced hyphal morphogenesis in C. albicans. Known or suspected permeases and transporters are indicated with light yellow boxes, while the dark yellow box displays the transcription factor CaStp2. Verified mechanisms of amino acid sensing, transport, and regulation are indicated by solid arrows, while proposed, suspected or so far not completely resolved connections are indicated by dashed arrows. Dotted arrows show the sensory activity of CaSsy1. The dotted circle represents a mitochondrion. Illustrated is the activation of the cAMP/PKA-pathway and hyphal induction via the CaMup1-mediated uptake of methionine and its subsequent conversion via SAM to polyamines, like spermine and spermidine. The exact mechanism for this remains unresolved. A second hyphal inducer is provided via the mitochondrial degradation of proline, resulting in the generation of a high amount of electron donors, which are subsequently used for ATP generation via oxidative phosphorylation. The resulting accumulation of ATP also leads to an activation of the cAMP/PKA pathway. This process is inhibited by high amounts of extracellular glucose through the blocking of mitochondrial activity. The uptake of arginine and proline which drives this process is at least partially independent from the SPS sensor system and could involve unidentified permeases or transporters. Amino aciddriven alkalinization represents the third described hyphae-inducing pathway. Extracellular amino acids are sensed via CaSsy1, leading to CaStp2-mediated expression of amino acid permeases. The amino acids are taken up and metabolized, creating large amounts of cytotoxic ammonium, which are then extruded by ammonium transporters such as CaAto5 and lead to alkalinization of the environmental $\mathrm{pH}$. This process results in hyphal morphogenesis, though the underlying mechanisms are not completely understood. CaGpr1 and CaGap1/2/6 are suspected to activate the CAMP/PKA pathway, although the nature of this activation and potential ligands have not been identified 
mitochondrial activity should be suppressed [22•]. It is likely that in this case, filamentation is mediated via CaDur1, 2 activity, which hydrolyzes the urea by-product of the arginine to proline conversion into ammonia and carbon dioxide. This process is reportedly also involved in hyphal morphogenesis and considered a slower, alternate way for arginine-induced filamentation $[19,22 \bullet]$.

Interesting in this context is a previous study, which linked the $\mathrm{G}$ protein-coupled receptor $\mathrm{CaGpr} 1$ to proline and methionine-induced hyphal morphogenesis dependent on the quality and quantity of the present carbon source [88, 89] (Fig. 1). In S. cerevisiae, ScGpr1 physically interacts with glucose, sucrose, and mannose, activating of the cAMP/PKA pathway $[90,91]$. In contrast, CaGpr1 appears dispensable for glucoseinduced cAMP increase, though low glucose levels seem to be a prerequisite for the $\mathrm{CaGpr} 1$ methionine interaction [88, 89]. It could be speculated that the CaGprl-mediated sensing of proline is necessary for the described hyphal morphogenesis, though it was shown to be SPS independent [22•]. However, the ligand(s) of $\mathrm{CaGpr} 1$ are not unequivocally identified and further research is required to elucidate CaGpr1-dependent signaling and its possible implications in arginine- and proline-induced filamentation [22•].

The $\mathrm{CnGpr} 4$ receptor in $C$. neoformans shows an activation pattern similar to $\mathrm{CaGpr} 1$, where it is internalized upon methionine exposure and required for methionine-induced elevation of cAMP, but is also dispensable for glucose-induced elevation. Nevertheless, a knockout mutant showed no attenuated virulence in a murine model [92]. Proline catabolism is also linked to $C$. neoformans virulence, since disruption of the catabolic pathway increased $C$. neoformans sensitivity to oxidative and nitrosative stresses, while a strain defective in proline catabolism had a mild defect in melanization and was avirulent in a murine model of intranasal infection [23•]. Like C. albicans, C. neoformans is also able to utilize proline as either a nitrogen and carbon source [23•, 87•]. However, in contrast to $C$. albicans, the corresponding catabolic enzymes are suppressed by primary nitrogen sources, pointing to NCRmediated regulation [23•].

Another mechanism for amino acid-induced filamentation in C. albicans is through intracellular methionine metabolism and the uptake of methionine from the host via CaMup1 [21•] (Fig. 1). Methionine is subsequently metabolized to polyamines, like spermine or spermidine, via decarboxylation of the intermediate S-adenosylmethionine (SAM). Ultimately, this leads to induction of the cAMP/PKA pathway and hyphal growth, but the mechanism of the polyamine-mediated PKA pathway induction is not fully understood. Further, spermine and spermidine can also drive hyphal morphogenesis independently of CaMup 1, likely via uptake through the characterized polyamine transporters CaDur3 and CaDur31 [93]. Another study showed that this pathway could be also triggered directly by the uptake of SAM via CaGap4 [65•]. Deletion of
CaMUP1 results in decreased virulence in a model of disseminated candidiasis, shortened hyphae, and reduced survival during confrontation with macrophages [21•]. Methionine's intertwined roles in cellular metabolism, signaling, and virulence underscores the importance of this amino acid for C. albicans.

The methionine permeases CnMup1 and CnMup3 of C. neoformans are also involved in virulence, since they are required for proper capsule formation [34]. In addition to methionine, $\mathrm{Cn} M U P 1$ and $\mathrm{Cn} M U P 3$ are induced by histidine and tryptophan and are NCR-regulated [34]. Aside from these two, the $C$. neoformans genome encodes for 8 additional amino acid permeases with broader specificity, named CnAAPI- 8 [56 ${ }^{\circ}$. In the presence of amino acids as the sole nitrogen source, $C n A A P 2 / 4 / 5 / 8$ were highly induced. Yet, with the exception of $\mathrm{Cn} A A P 4$, their expression was repressed upon the addition of ammonium sulfate, pointing to NCR-mediated regulation [56]. CnAap4 and CnAap5 are highly similar and to some degree redundant, as single knockout mutants still showed full virulence in a murine model, while a double knockout was avirulent [34]. Further, the double mutant is impaired in growth on every amino acid as a nitrogen source at $37^{\circ} \mathrm{C}$ [34]. It could be speculated that the presence of both permeases represents a gene duplication that facilitated adaptation to the environment/host, since only CnAap5 is regulated by NCR. Recently, it was reported that the double knockout of CnAAP1 and CnAAP2 shows impaired growth on multiple amino acids as nitrogen sources and affects capsulation [35]. Interestingly, $\mathrm{Cn} A A P 1 / 2 / 4 / 5 / 6 / 8$ are induced in the presence of the unfavorable carbon source, galactose [56]. It is therefore tempting to speculate that this represents a potential stimulus for utilization of amino acids as a carbon source in glucoselimited environments. Nevertheless, the regulatory pathways mediating amino acid sensing in vivo remain poorly understood and need to be investigated further.

For A. fumigatus, nearly nothing is known about the activity and role of AAPs. The genome encodes at least one ScGAP1 ortholog as well as multiple other predicted permeases. Transcriptome analyses suggest that amino acid uptake might play a role during infection, since amino acid metabolism is differentially regulated in confrontation to platelets in vitro and in whole blood $[94,95]$. Furthermore, a predicted amino acid transporter was found to be upregulated in conidia confronted with human airway epithelia cells [96]. AAPs are also upregulated in the presence of proteins as a nitrogen source, suggesting the A. fumigatus degrades host proteins to scavenge for nutrients [83]. Indeed, genes involved in amino acid catabolism, including 13 AAPs and various secreted proteases, are induced during invasive aspergillosis, while amino acid biosynthetic pathways are downregulated [97]. It is hypothesized that this represents a clear starvation response, triggering the induction of genes regulating the utilization of alternative nutrient sources found in the lung. This is supported 
by the concept that $A$. fumigatus relies heavily on the amino acids' valine, isoleucine, and methionine as carbon sources during invasive aspergillosis [98••]. This study proposes that the catabolism of these amino acids leads to the accumulation of propionyl-CoA, which is incorporated as pyruvate into the primary metabolism via the methylcitrate cycle-a pathway closely linked to the TCA cycle. Since disruption of methylcitrate synthase, a key element of the methylcitrate cycle, results in enhanced clearance by macrophages in vitro and strongly attenuated virulence in a murine model of invasive aspergillosis, it is hypothesized that acquisition of amino acids is important for A. fumigatus virulence [98••].

\section{Amino Acid-Driven Alkalinization of the Environmental pH}

The neutralization of the environmental $\mathrm{pH}$, through the conversion of urea to carbon dioxide and ammonia by secreted ureases, is a well-known virulence factor in numerous plant and human pathogenic fungi [99]. For example, the ureolytic activity of $C$. neoformans modulates the $\mathrm{pH}$ of the macrophage phagolysosome, thereby affecting non-lytic exocytosis and intracellular replication of the yeast [100•]. In contrast, C. albicans does not encode a secreted urease. In this fungus, free ammonium is generated during arginine catabolism to ornithine and urea, and then subsequently processed via the intracellular enzyme urea amidolyase CaDur1, 2 to carbon dioxide and ammonia-both potent inducers of hyphal growth [101]. To balance out the potentially toxic quantity of ammonium ions in the cell, C. albicans has evolved a family of 10 ammonium transporters (ATOs), CaAto1-10 [102]. Although in a recent study, Danhof and Lorenz showed that deletion of $\mathrm{CaATO5}$ or the expression of a dominant-negative $\mathrm{CaATO1}{ }^{G 53 D}$ allele resulted in reduced ammonium release from the cell, the exact role of the ATOs is not understood [102]. Interestingly, despite the low enzymatic activity of CaDur1, 2, C. albicans alkalinizes the environmental $\mathrm{pH}$ with remarkable speed-in vitro growth on amino acids as the sole carbon source raises the environmental $\mathrm{pH}$ from 4.0 to neutral within $6-8 \mathrm{~h}$ [19]. This process is coordinated by CaStp2, which activation triggers the expression of most AAPs, OPTs, and ATOs along with amino acid metabolism genes, and results in amino acid uptake, ammonium extrusion, and hyphal morphogenesis [25•] (Fig. 1). Interestingly, the canonical regulator for $\mathrm{pH}$ adaptation and $\mathrm{pH}$-induced hyphal morphogenesis, CaRim101, is dispensable for alkalinization [19], and alkalinization-driven hyphal morphogenesis (own unpublished data).

Environmental alkalinization can be observed upon in vitro growth in medium mimicking the nutrient composition of whole saliva and vaginal secretions in humans, suggesting that C. albicans could modulate the $\mathrm{pH}$ within the host [25•].
However, the contribution of this phenomenon to commensalism and/or pathogenicity in the oral cavity and vulvovaginal area remains unclear due to the lack of a suitable animal model that could represent the nutrient and $\mathrm{pH}$ conditions of the human host.

A few studies have demonstrated the importance of environmental neutralization in fungal escape from the macrophage phagosome. Indeed, the phagosome is hypothesized to resemble a glucose poor, but amino acid-enriched environment, which is evidenced by the fact that CaSsy1, CaStp2, and other proteins involved in the amino acid-driven alkalinization are activated upon phagocytosis and their absence renders the cell incapable of phagosomal escape due to failure to neutralize the phagosome [25•, 26, 102-104]. A recent study demonstrated that hyphal morphogenesis ruptures the phagosomal membrane, leading to luminal alkalinization, and contradicting the model of alkalinization as the cause of hyphal morphogenesis in the phagosome [105•]. Nevertheless, mutants defective in amino acid sensing also fail in filamentous growth, likely due to an inability to meet the growing energy needs of the cell following phagocytosis. The slower/impaired morphogenesis could allow the immune cell to repair the phagosomal membrane and prevent alkalinization. Further research is required to understand in greater detail the reason(s) for $C$. albicans hyphal morphogenesis within the macrophage phagosome.

Another observation worth discussing is that upon C. albicans phagocytosis, genes required for arginine biosynthesis are specifically upregulated [106]. This induction appears to be independent of $\mathrm{CaGn} 4$, a transcription factor that controls amino acid biosynthesis in response to starvation, but dependent on sublethal concentrations of reactive oxygen species (ROS) [48, 106]. Although the underlying mechanism behind this is not fully understood, it is evident that strains lacking key biosynthetic genes are defective in filamentation during phagocytosis [106].

\section{Amino Acid Metabolism as a Potential Drug Target}

Components of the amino acid metabolism represent an intriguing and novel target for antifungal therapy, since there are a variety of primary fungal metabolic pathways that are absent in the host [107]. This is particularly the case for the biosynthesis of 10 amino acids which are at least semi-essential for humans: Ile, Leu, Lys, Met, Phe, Thr, Trp, Val, Arg, and His. The potential of essential amino acid biosynthetic pathways as antifungal targets has been summarized previously [24, 108]. Another option would be targeting or exploiting fungal amino acid transport mechanisms. A current review of this strategy can be found here [109].

In contrast to what has been reported for $C$. neoformans and A. fumigatus, only a few reports exist concerning amino 
acid biosynthetic pathways essential for virulence in C. albicans [24, 108]. For example, the disruption of the threonine and branched-chain amino acid biosynthetic pathways result in attenuated virulence in intravenous infection model in mice $[110,111]$. It could be speculated that through C. albicans' adaptation to the human body as a commensal, it evolved sophisticated and specialized amino acid uptake systems to guarantee efficient supply and utilization within the host environment. Consequently, it could be more promising to target the responsible sensing and transport mechanisms in C. albicans, which lack human orthologs.

\section{Conclusion and Future Perspectives}

Amino acid utilization is essential for survival and infectivity of pathogenic fungi within the host. Yet, the factors and processes controlling amino acid-associated metabolism and virulence remain poorly understood to date. While the nature of amino acid uptake is partially described for C. albicans and C. neoformans, it is uncharacterized for A. fumigatus. Although the genome of the latter organism encodes for over a hundred secreted proteases, the functionality and specificity of only a few has been reported with no specific phenotypes, likely due to their redundant and overlapping functions. In addition, while strong evidence for the assimilation of carbon from valine, isoleucine, and methionine via the methylcitrate cycle during pulmonary aspergillosis exists, exactly how these extracellular amino acids are sensed and internalized remains elusive [98••]. Furthermore, the absence of orthologs for the SPS sensor system is striking and suggests either a more basal and nonspecific uptake mechanism, likely via a ScGap1 ortholog or a so far unidentified regulatory circuit.

Similar questions arise for C. neoformans, whose mechanisms for the sensing and uptake of extracellular amino acids have not yet been described. Further, the role of amino acid metabolism in infection remains unclear, though initial observations have been made [34]. Similarly, the role of amino acids in C. albicans morphogenesis, A. fumigatus melanization, and $C$. neoformans capsule formation remains to be investigated.

Due to the ability of $C$. albicans to assimilate different carbon sources simultaneously, it is tempting to speculate that a unique rewiring of NCR-mediated regulation of amino acid metabolism could provide similar advantages regarding nitrogen assimilation in this fungus [17]. First observations in this direction have already been made [87•], and a detailed investigation of these regulatory pathways could help in better understanding the nutritional basis of $C$. albicans colonization and virulence. A recent study showed differences in amino acid metabolism in C. albicans biofilms, with a strong upregulation of arginine, proline, aspartate, and glutamate metabolism in high biofilm-forming isolates, while low biofilm- forming isolates displayed an upregulation in purine, starch, and sucrose metabolism, suggesting that the ability to better metabolize amino acids leads to a growth advantage in nutrient-poor environments [20•]. However, the metabolic consequences arising from these differences and their outcome for the fungus, as well as the underlying regulatory systems, are unknown and require further investigations.

Altogether, amino acid metabolism drives growth and virulence of fungal pathogens within the host and further investigations are required to gain a more comprehensive understanding of the infection-relevant processes and their potential use as antifungal targets.

Acknowledgments We thank our colleagues Amelia Barber, Bettina Böttcher, and Franziska Gerwien from the ZIK Septomics for the critical reading of the manuscript and fruitful discussions.

Funding Statement This work was supported by the German Federal Ministry of Education and Research (BMBF grant no. 03Z22JN11) to SV.

\section{Compliance with Ethical Standards}

Conflict of Interest Enrico Garbe and Slavena Vylkova each declare no potential conflicts of interest.

Human and Animal Rights and Informed Consent This article does not contain any studies with human or animal subjects performed by any of the authors.

Disclaimer The funders had no role in study design, data collection and analysis, decision to publish, or preparation of the manuscript.

Open Access This article is distributed under the terms of the Creative Commons Attribution 4.0 International License (http:// creativecommons.org/licenses/by/4.0/), which permits unrestricted use, distribution, and reproduction in any medium, provided you give appropriate credit to the original author(s) and the source, provide a link to the Creative Commons license, and indicate if changes were made.

\section{References}

Papers of particular interest, published recently, have been highlighted as:

- Of importance

•• Of major importance

1. Brown GD, Denning DW, Gow NAR, Levitz SM, Netea MG, White TC. Hidden killers: human fungal infections. Sci Transl Med. 2012;4(165). https://doi.org/10.1126/scitranslmed.3004404.

2. Sudbery PE. Growth of Candida albicans hyphae. Nat Rev Microbiol. 2011;9(10):737-48. https://doi.org/10.1038/ nrmicro2636.

3. Noble SM, Gianetti BA, Witchley JN. Candida albicans cell-type switching and functional plasticity in the mammalian host. Nat Rev Microbiol. 2016;15(2):96-108. https://doi.org/10.1038/ nrmicro.2016.157. 
4. Lohse MB, Gulati M, Johnson AD, Nobile CJ. Development and regulation of single- and multi-species Candida albicans biofilms. Nat Rev Microbiol. 2017;16(1):19-31. https://doi.org/10.1038/ nrmicro.2017.107.

5. Brown AJ, Brown GD, Netea MG, Gow NA. Metabolism impacts upon Candida immunogenicity and pathogenicity at multiple levels. Trends Microbiol. 2014;22(11):614-22. https://doi.org/ 10.1016/j.tim.2014.07.001.

6. Brown AJ, Budge S, Kaloriti D, Tillmann A, Jacobsen MD, Yin Z, et al. Stress adaptation in a pathogenic fungus. J Exp Biol. 2014;217(Pt 1):144-55. https://doi.org/10.1242/jeb.088930.

7. van de Veerdonk FL, Gresnigt MS, Romani L, Netea MG, Latge JP. Aspergillus fumigatus morphology and dynamic host interactions. Nat Rev Microbiol. 2017;15(11):661-74. https://doi.org/10. 1038/nrmicro.2017.90.

8. Krappmann S. How to invade a susceptible host: cellular aspects of aspergillosis. Curr Opin Microbiol. 2016;34:136-46. https:// doi.org/10.1016/j.mib.2016.10.002.

9. Amich J, Krappmann S. Deciphering metabolic traits of the fungal pathogen Aspergillus fumigatus: redundancy vs. essentiality. Front Microbiol. 2012;3(414). https://doi.org/10.3389/fmicb.2012. 00414.

10. Kronstad JW, Attarian R, Cadieux B, Choi J, D'Souza CA, Griffiths EJ, et al. Expanding fungal pathogenesis: Cryptococcus breaks out of the opportunistic box. Nat Rev Microbiol. 2011;9(3):193-203. https://doi.org/10.1038/nrmicro2522.

11. Kronstad J, Saikia S, Nielson ED, Kretschmer M, Jung W, Hu G, et al. Adaptation of Cryptococcus neoformans to mammalian hosts: integrated regulation of metabolism and virulence. Eukaryot Cell. 2012;11(2):109-18. https://doi.org/10.1128/EC. 05273-11.

12. Ballou ER, Johnston SA. The cause and effect of Cryptococcus interactions with the host. Curr Opin Microbiol. 2017;40:88-94. https://doi.org/10.1016/j.mib.2017.10.012.

13. Dambuza IM, Drake T, Chapuis A, Zhou X, Correia J, TaylorSmith L, et al. The Cryptococcus neoformans Titan cell is an inducible and regulated morphotype underlying pathogenesis. PLoS Pathog. 2018;14(5):e1006978. https://doi.org/10.1371/ journal.ppat.1006978.

14. Owen DH, Katz DF. A vaginal fluid simulant. Contraception. 1999;59(2):91-5. https://doi.org/10.1016/S0010-7824(99)00010-4.

15. Wong L, Sissions CH. A comparison of human dental plaque microcosm biofilms grown in an undefined medium and a chemically defined artificial saliva. Arch Oral Biol. 2001;46(6):477-86. https://doi.org/10.1016/S0003-9969(01)00016-4.

16. Brosnan JT. Interorgan amino acid transport and its regulation. J Nutr. 2003;133(6):2068S-72S. https://doi.org/10.1093/jn/133.6. 2068S.

17. Sandai D, Zhikang Y, Selway L, Stead D, Walker J, Leach MD, et al. The evolutionary rewiring of ubiquitination targets has reprogrammed the regulation of carbon assimilation in the pathogenic yeast Candida albicans. MBio. 2012;3(6):e00495-12. https://doi.org/10.1128/mBio.00495-12.

18. Pérez JC, Kumamoto CA, Johnson AD. Candida albicans commensalism and pathogenicity are intertwined traits directed by a tightly knit transcriptional regulatory circuit. PLoS Biol. 2013;11(3):e1001510. https://doi.org/10.1371/journal.pbio. 1001510 .

19. Vylkova S, Carman AJ, Danhof HA, Collette JR, Zhou H, Lorenz MC. The fungal pathogen Candida albicans autoinduces hyphal morphogenesis by raising extracellular pH. MBio. 2011;2(3): e00055-11. https://doi.org/10.1128/mBio.00055-11.

20. Rajendran R, May A, Sherry L, Kean R, Williams C, Jones BL, et al. Integrating Candida albicans metabolism with biofilm heterogeneity by transcriptome mapping. Sci Rep. 2016;6:35436. https://doi.org/10.1038/srep35436 This study depicts differences in the biofilm-forming behavior of $\mathrm{C}$. albicans isolates and presents a highly upregulated amino acid metabolism in high biofilm-forming isolates.

21. Schrevens S, Van Zeebroeck G, Riedelberger M, Tournu H, Kuchler K, Van Dijck P. Methionine is required for cAMPPKA-mediated morphogenesis and virulence of Candida albicans. Mol Microbiol. 2018;108(3):258-75. https://doi.org/ $10.1111 / \mathrm{mmi} .13933$ In this study, the hyphal morphogenesis of C. albicans via uptake of methionine and subsequent conversion to polyamines is described.

22. Silao FGS, Ward M, Ryman K, Wallstrom A, Brindefalk B, Udekwu K, et al. Mitochondrial proline catabolism activates Ras1/cAMP/PKA-induced filamentation in Candida albicans. PLoS Genet. 2019;15(2):e1007976. https://doi.org/10.1371/ journal.pgen.1007976 This study describes the hyphal morphogenesis in $C$. albicans via the mitochondrial catabolism of proline, triggering the cAMP/PKA pathway. This represents the first resolved mechanism of amino acidinduced PKA activation.

23. Lee IR, Lui EY, Chow EW, Arras SD, Morrow CA, Fraser JA. Reactive oxygen species homeostasis and virulence of the fungal pathogen Cryptococcus neoformans requires an intact proline catabolism pathway. Genetics. 2013;194(2):421-33. https://doi.org/ 10.1534 /genetics.113.150326 In this study, the critical role of an intact proline catabolism for $\mathrm{C}$. neoformans virulence is described.

24. Amich J, Bignell E. Amino acid biosynthetic routes as drug targets for pulmonary fungal pathogens: what is known and why do we need to know more? Curr Opin Microbiol. 2016;32:151-8. https:// doi.org/10.1016/j.mib.2016.06.003.

25. Vylkova S, Lorenz MC. Modulation of phagosomal $\mathrm{pH}$ by Candida albicans promotes hyphal morphogenesis and requires Stp2p, a regulator of amino acid transport. PLoS Pathog. 2014;10(3):e1003995. https://doi.org/10.1371/journal.ppat. 1003995 First report about alkalinization of macrophage phagosomes by $\mathrm{C}$. albicans leading to phagosomal escape and its dependency on functional amino acid sensing.

26. Miramon P, Lorenz MC. The SPS amino acid sensor mediates nutrient acquisition and immune evasion in Candida albicans. Cell Microbiol. 2016;18(11):1611-24. https://doi.org/10.1111/cmi. 12600 .

27. Limjindaporn T, Khalaf RA, Fonzi WA. Nitrogen metabolism and virulence of Candida albicans require the GATA-type transcriptional activator encoded by GAT1. Mol Microbiol. 2003;50(3): 993-1004. https://doi.org/10.1046/j.1365-2958.2003.03747.x.

28. Liao WL, Ramon AM, Fonzi WA. GLN3 encodes a global regulator of nitrogen metabolism and virulence of $C$. albicans. Fungal Genet Biol. 2008;45(4):514-26. https://doi.org/10.1016/j.fgb.2007.08. 006.

29. Kmetzsch L, Staats CC, Simon E, Fonseca FL, Oliveira DL, Joffe LS, et al. The GATA-type transcriptional activator Gat1 regulates nitrogen uptake and metabolism in the human pathogen Cryptococcus neoformans. Fungal Genet Biol. 2011;48(2):1929. https://doi.org/10.1016/j.fgb.2010.07.011.

30. Hensel M, Arst HN, Aufauvre-Brown A, Holden DW. The role of the Aspergillus fumigatus areA gene in invasive pulmonary aspergillosis. Mol Gen Genet. 1998;258(5):553-7. https://doi.org/10. 1007/s004380050767

31. Lee H, Khanal Lamichhane A, Garraffo HM, Kwon-Chung KJ, Chang YC. Involvement of PDK1, PKC and TOR signalling pathways in basal fluconazole tolerance in Cryptococcus neoformans. Mol Microbiol. 2012;84(1):130-46. https://doi.org/10.1111/j. 1365-2958.2012.08016.x.

32. Brand A, MacCallum DM, Brown AJP, Gow NAR, Odds FC. Ectopic expression of URA3 can influence the virulence phenotypes and proteome of Candida albicans but can be overcome by 
targeted reintegration of URA3 at the RPS10 Locus. Eukaryot Cell. 2004;3(4):900-9. https://doi.org/10.1128/EC.3.4.900-909. 2004.

33. Krappmann S, Bignell EM, Reichard U, Rogers T, Haynes K, Braus GH. The Aspergillus fumigatus transcriptional activator $\mathrm{CpcA}$ contributes significantly to the virulence of this fungal pathogen. Mol Microbiol. 2004;52(3):785-99. https://doi.org/10.1111/ j.1365-2958.2004.04015.x.

34. Martho KF, de Melo AT, Takahashi JP, Guerra JM, Santos DC, Purisco SU, et al. Amino acid permeases and virulence in Cryptococcus neoformans. PLoS One. 2016;11(10):e0163919. https://doi.org/10.1371/journal.pone.0163919.

35. Calvete CL, Martho KF, Felizardo G, Paes A, Nunes JM, Ferreira $\mathrm{CO}$, et al. Amino acid permeases in Cryptococcus neoformans are required for high temperature growth and virulence; and are regulated by Ras signaling. PLoS One. 2019;14(1):e0211393. https:// doi.org/10.1371/journal.pone.0211393.

36. Zhang W, Du G, Zhou J, Chen J. Regulation of sensing, transportation, and catabolism of nitrogen sources in Saccharomyces cerevisiae. Microbiol Mol Biol Rev. 2018;82(1):e00040-17. https://doi.org/10.1128/MMBR.00040-17.

37. Ries LNA, Beattie S, Cramer RA, Goldman GH. Overview of carbon and nitrogen catabolite metabolism in the virulence of human pathogenic fungi. Mol Microbiol. 2018;107(3):277-97. https://doi.org/10.1111/mmi.13887.

38. Hofman-Bang J. Nitrogen catabolite repression in Saccharomyces cerevisiae. Mol Biotechnol. 1999;12(1):35-73. https://doi.org/10. 1385/MB:12:1:35.

39. Magasanik B, Kaiser CA. Nitrogen regulation in Saccharomyces cerevisiae. Gene. 2002;290(1-2):1-18. https://doi.org/10.1016/ S0378-1119(02)00558-9.

40. Dabas N, Morschhäuser J. Control of ammonium permease expression and filamentous growth by the GATA transcription factors GLN3 and GAT1 in Candida albicans. Eukaryot Cell. 2007;6(5):875-88. https://doi.org/10.1128/EC.00307-06.

41. Lee IR, Chow EW, Morrow CA, Djordjevic JT, Fraser JA. Nitrogen metabolite repression of metabolism and virulence in the human fungal pathogen Cryptococcus neoformans. Genetics. 2011;188(2):309-23. https://doi.org/10.1534/genetics.111. 128538.

42. Loewith R, Hall MN. Target of rapamycin (TOR) in nutrient signaling and growth control. Genetics. 2011;189(4):1177-201. https://doi.org/10.1534/genetics.111.133363.

43. Bastidas RJ, Heitman J, Cardenas ME. The protein kinase Tor1 regulates Adhesin gene expression in Candida albicans. PLoS Pathog. 2009;5(2):e1000294. https://doi.org/10.1371/journal.ppat.1000294.

44. Baldin C, Valiante V, Krüger T, Schafferer L, Haas H, Kniemeyer $\mathrm{O}$, et al. Comparative proteomics of a tor inducible Aspergillus fumigatus mutant reveals involvement of the Tor kinase in iron regulation. Proteomics. 2015;15(13):2230-43. https://doi.org/10. 1002/pmic.201400584.

45. Böttcher B, Pöllath C, Staib P, Hube B, Brunke S. Candida species rewired hyphae developmental programs for chlamydospore formation. Front Microbiol. 2016;7:1697. https://doi.org/10.3389/ fmicb.2016.01697.

46. Cruz MC, Cavallo LM, Görlach JM, Cox G, Perfect JR, Cardenas ME, et al. Rapamycin antifungal action is mediated via conserved complexes with FKBP12 and TOR kinase homologs in Cryptococcus neoformans. Mol Cell Biol. 1999;19(6):4101-12.

47. Hinnebusch AG. Translational regulation of GCN4 and the general amino acid control of yeast. Annu Rev Microbiol. 2005;59(1): 407-50. https://doi.org/10.1146/annurev.micro.59.031805. 133833.

48. Tripathi G, Wiltshire C, Macaskill S, Tournu H, Budge S, Brown AJP. Gcn 4 co-ordinates morphogenetic and metabolic responses to amino acid starvation in Candida albicans. EMBO J. 2002;21(20):5448-56. https://doi.org/10.1093/emboj/cdf507.

49. Ljungdahl PO. Amino-acid-induced signalling via the SPSsensing pathway in yeast. Biochem Soc Trans. 2009;37(1):2427. https://doi.org/10.1042/BST0370242.

50. Ljungdahl PO, Gimeno CJ, Styles CA, Fink GR. SHR3: A novel component of the secretory pathway specifically required for localization of amino acid permeases in yeast. Cell. 1992;71(3): 463-78. https://doi.org/10.1016/0092-8674(92)90515-E.

51. Gilstring CF, Melin-Larsson M, Ljungdahl PO. Shr3p mediates specific COPII coatomer-cargo interactions required for the packaging of amino acid permeases into ER-derived transport vesicles. Mol Biol Cell. 1999;10(11):3549-65. https://doi.org/10.1091/ mbc.10.11.3549.

52. Brega E, Zufferey R, Mamoun CB. Candida albicans Csylp is a nutrient sensor important for activation of amino acid uptake and hyphal morphogenesis. Eukaryot Cell. 2004;3(1):135-43. https:// doi.org/10.1128/ec.3.1.135-143.2004.

53. Martinez P, Ljungdahl PO. Divergence of Stp1 and Stp2 transcription factors in Candida albicans places virulence factors required for proper nutrient acquisition under amino acid control. Mol Cell Biol. 2005;25(21):9435-46. https://doi.org/10.1128/MCB.25.21. 9435-9446.2005.

54. Martinez P, Ljungdahl PO. An ER packaging chaperone determines the amino acid uptake capacity and virulence of Candida albicans. Mol Microbiol. 2004;51(2):371-84. https://doi.org/10. 1046/j.1365-2958.2003.03845.x.

55. Ramachandra S, Linde J, Brock M, Guthke R, Hube B, Brunke S. Regulatory networks controlling nitrogen sensing and uptake in Candida albicans. PLoS One. 2014;9(3):e92734. https://doi.org/ 10.1371/journal.pone.0092734.

56. Fernandes JD, Martho K, Tofik V, Vallim MA, Pascon RC. The role of amino acid permeases and tryptophan biosynthesis in Cryptococcus neoformans survival. PLoS One. 2015;10(7): e0132369. https://doi.org/10.1371/journal.pone.0132369 First description and characterization of amino acid permeases in C. neoformans.

57. Jauniaux J-C, Grenson M. GAP1, the general amino acid permease gene of Saccharomyces cerevisiae. Eur J Biochem. 1990;190(1):39-44. https://doi.org/10.1111/j.1432-1033.1990. tb15542.x.

58. Uemura T, Kashiwagi K, Igarashi K. Uptake of putrescine and spermidine by Gaplp on the plasma membrane in Saccharomyces cerevisiae. Biochem Biophys Res Commun. 2005;328(4):1028-33. https://doi.org/10.1016/j.bbrc.2005.01. 064.

59. Van Zeebroeck G, Rubio-Texeira M, Schothorst J, Thevelein JM. Specific analogues uncouple transport, signalling, oligoubiquitination and endocytosis in the yeast Gap1 amino acid transceptor. Mol Microbiol. 2014;93(2):213-33. https://doi.org/ 10.1111/mmi.12654.

60. Donaton MCV, Holsbeeks I, Lagatie O, Van Zeebroeck G, Crauwels M, Winderickx J, et al. The Gapl general amino acid permease acts as an amino acid sensor for activation of protein kinase A targets in the yeast Saccharomyces cerevisiae. Mol Microbiol. 2003;50(3):911-29. https://doi.org/10.1046/j.13652958.2003.03732.x.

61. Van Zeebroeck G, Bonini BM, Versele M, Thevelein JM. Transport and signaling via the amino acid binding site of the yeast Gap1 amino acid transceptor. Nat Chem Biol. 2009;5(1): 45-52. https://doi.org/10.1038/nchembio.132.

62. Voordeckers K, Kimpe M, Haesendonckx S, Louwet W, Versele M, Thevelein JM. Yeast 3-phosphoinositide-dependent protein kinase-1 (PDK1) orthologs Pkh1-3 differentially regulate phosphorylation of protein kinase A (PKA) and the protein kinase $\mathrm{B}$ 
(PKB)/S6K ortholog Sch9. J Biol Chem. 2011;286(25):2201727. https://doi.org/10.1074/jbc.M110.200071.

63. Biswas S, Roy M, Datta A. $N$-acetylglucosamine-inducible CaGAPl encodes a general amino acid permease which coordinates external nitrogen source response and morphogenesis in Candida albicans. Microbiology. 2003;149(9):2597-608. https://doi.org/10.1099/mic.0.26215-0.

64. Kraidlova L, Van Zeebroeck G, Van Dijck P, Sychrova H. The Candida albicans GAP gene family encodes permeases involved in general and specific amino acid uptake and sensing. Eukaryot Cell. 2011;10(9):1219-29. https://doi.org/10.1128/EC.05026-11.

65. Kraidlova L, Schrevens S, Tournu H, Van Zeebroeck G, Sychrova H, Van Dijck P. Characterization of the Candida albicans amino acid permease family: Gap2 is the only general amino acid permease and Gap4 is an S-adenosylmethionine (SAM) transporter required for SAM-induced morphogenesis. mSphere. 2016;1(6): e00284-16. https://doi.org/10.1128/mSphere.00284-16 Complete characterization of the C. albicans ScGap1 orthologs and identification of CaGap2 as the functional ortholog

66. Monod M, Capoccia S, Léchenne B, Zaugg C, Holdom M, Jousson O. Secreted proteases from pathogenic fungi. Int J Med Microbiol. 2002;292(5-6):405-19. https://doi.org/10.1078/14384221-00223.

67. Naglik JR, Challacombe SJ, Hube B. Candida albicans secreted aspartyl proteinases in virulence and pathogenesis. Microbiol Mol Biol Rev. 2003;67(3):400-28. https://doi.org/10.1128/MMBR.67. 3.400-428.2003.

68. Reuß O, Morschhäuser J. A family of oligopeptide transporters is required for growth of Candida albicans on proteins. Mol Microbiol. 2006;60(3):795-812. https://doi.org/10.1111/j.13652958.2006.05136.x.

69. Hube B, Sanglard D, Odds FC, Hess D, Monod M, Schäfer W, et al. Disruption of each of the secreted aspartyl proteinase genes SAP1, SAP2, and SAP3 of Candida albicans attenuates virulence. Infect Immun. 1997;65(9):3529-38.

70. Sanglard D, Hube B, Monod M, Odds FC, Gow NA. A triple deletion of the secreted aspartyl proteinase genes SAP4, SAP5, and SAP6 of Candida albicans causes attenuated virulence. Infect Immun. 1997;65(9):3539-46.

71. Salkowski CA, Balish E. Cutaneous cryptococcosis in athymic and beige-athymic mice. Infect Immun. 1991;59(5):1785-9.

72. Vu K, Tham R, Uhrig JP, Thompson GR, Na Pombejra S, Jamklang M, et al. Invasion of the central nervous system by Cryptococcus neoformans requires a secreted fungal metalloprotease. MBio. 2014;5(3):e01101-14. https://doi.org/10. 1128/mBio.01101-14.

73. Xu C-Y, Zhu H-M, Wu J-H, Wen H, Liu C-J. Increased permeability of blood-brain barrier is mediated by serine protease during Cryptococcus meningitis. J Int Med Res. 2014;42(1):85-92. https://doi.org/10.1177/0300060513504365.

74. Chen LC, Blank ES, Casadevall A. Extracellular proteinase activity of Cryptococcus neoformans. Clin Diagn Lab Immunol. 1996;3(5):570-4.

75. Clarke SC, Dumesic PA, Homer CM, O'Donoghue AJ, La Greca F, Pallova L, et al. Integrated activity and genetic profiling of secreted peptidases in Cryptococcus neoformans reveals an aspartyl peptidase required for low $\mathrm{pH}$ survival and virulence. PLoS Pathog. 2016;12(12):e1006051. https://doi.org/10.1371/ journal.ppat.1006051.

76. Wiles AM, Naider F, Becker JM. Transmembrane domain prediction and consensus sequence identification of the oligopeptide transport family. Res Microbiol. 2006;157(4):395-406. https:// doi.org/10.1016/j.resmic.2005.10.004

77. Homer CM, Summers DK, Goranov AI, Clarke SC, Wiesner DL, Diedrich JK, et al. Intracellular action of a secreted peptide required for fungal virulence. Cell Host Microbe. 2016;19(6): 849-64. https://doi.org/10.1016/j.chom.2016.05.001.

78. Gifford AHT, Klippenstein JR, Moore MM. Serum stimulates growth of and proteinase secretion by Aspergillus fumigatus. Infect Immun. 2002;70(1):19-26. https://doi.org/10.1128/IAI.70. 1.19-26.2002.

79. Nierman WC, Pain A, Anderson MJ, Wortman JR, Kim HS, Arroyo J, et al. Genomic sequence of the pathogenic and allergenic filamentous fungus Aspergillus fumigatus. Nature. 2005;438(7071):1151-6. https://doi.org/10.1038/nature04332.

80. Sharon H, Hagag S, Osherov N. Transcription factor PrtT controls expression of multiple secreted proteases in the human pathogenic mold Aspergillus fumigatus. Infect Immun. 2009;77(9):4051-60. https://doi.org/10.1128/IAI.00426-09.

81. Bergmann A, Hartmann T, Cairns T, Bignell EM, Krappmann S. A regulator of Aspergillus fumigatus extracellular proteolytic activity is dispensable for virulence. Infect Immun. 2009;77(9): 4041-50. https://doi.org/10.1128/IAI.00425-09.

82. Farnell E, Rousseau K, Thornton DJ, Bowyer P, Herrick SE. Expression and secretion of Aspergillus fumigatus proteases are regulated in response to different protein substrates. Fungal Biol. 2012;116(9):1003-12. https://doi.org/10.1016/j.funbio.2012.07.004.

83. Hartmann T, Cairns TC, Olbermann P, Morschhäuser J, Bignell EM, Krappmann S. Oligopeptide transport and regulation of extracellular proteolysis are required for growth of Aspergillus fumigatus on complex substrates but not for virulence. Mol Microbiol. 2011;82(4):917-35. https://doi.org/10.1111/j.13652958.2011.07868.x.

84. Namvar S, Warn P, Farnell E, Bromley M, Fraczek M, Bowyer P, et al. Aspergillus fumigatus proteases, Asp f 5 and Asp f 13, are essential for airway inflammation and remodelling in a murine inhalation model. Clin Exp Allergy. 2015;45(5):982-93. https:// doi.org/10.1111/cea.12426.

85. Didion T, Birgitte R, Jørgensen MU, Kielland-Brandt MC, Andersen HA. The permease homologue Ssylp controls the expression of amino acid and peptide transporter genes in Saccharomyces cerevisiae. Mol Microbiol. 1998;27(3):643-50. https://doi.org/10.1046/j.1365-2958.1998.00714.x.

86. Kodama Y, Omura F, Takahashi K, Shirahige K, Ashikari TJCG. Genome-wide expression analysis of genes affected by amino acid sensor Ssy1p in Saccharomyces cerevisiae. Curr Genet. 2002;41(2):63-72. https://doi.org/10.1007/s00294-002-0291-1.

87. Tebung WA, Omran RP, Fulton DL, Morschhauser J, Whiteway M. Put3 positively regulates proline utilization in Candida albicans. mSphere. 2017;2(6):e00354-17. https://doi.org/10. $1128 / \mathrm{mSphere} .00354-17$ Reports that C. albicans is able to utilize proline as either a carbon or nitrogen source independent of NCR-mediated regulation.

88. Maidan MM, De Rop L, Serneels J, Exler S, Rupp S, Tournu H, et al. The G protein-coupled receptor Gpr1 and the Galpha protein Gpa2 act through the cAMP-protein kinase A pathway to induce morphogenesis in Candida albicans. Mol Biol Cell. 2005;16(4): 1971-86. https://doi.org/10.1091/mbc.e04-09-0780.

89. Maidan MM, Thevelein JM, Van Dijck P. Carbon source induced yeast-to-hypha transition in Candida albicans is dependent on the presence of amino acids and on the G-protein-coupled receptor Gpr1. Biochem Soc Trans. 2005;33:291-3. https://doi.org/10. 1042/BST0330291.

90. Lemaire K, Van de Velde S, Van Dijck P, Thevelein JM. Glucose and sucrose act as agonist and mannose as antagonist ligands of the G protein-coupled receptor Gpr1 in the yeast Saccharomyces cerevisiae. Mol Cell. 2004;16(2):293-9. https://doi.org/10.1016/j. molcel.2004.10.004.

91. Yun C-W, Tamaki H, Nakayama R, Yamamoto K, Kumagai H. Gprlp, a putative G-protein coupled receptor, regulates glucosedependent cellular cAMP level in yeast Saccharomyces 
cerevisiae. Biochem Biophys Res Commun. 1998;252(1):29-33. https://doi.org/10.1006/bbrc.1998.9600.

92. Xue C, Bahn Y-S, Cox GM, Heitman J. G protein-coupled receptor Gpr4 senses amino acids and activates the cAMP-PKA pathway in Cryptococcus neoformans. Mol Biol Cell. 2006;17(2): 667-79. https://doi.org/10.1091/mbc.e05-07-0699.

93. Kumar R, Chadha S, Saraswat D, Bajwa JS, Li RA, Conti HR, et al. Histatin 5 uptake by Candida albicans utilizes polyamine transporters Dur3 and Dur31 proteins. J Biol Chem. 2011;286(51):43748-58. https://doi.org/10.1074/jbc.M111. 311175.

94. Perkhofer S, Zenzmaier C, Frealle E, Blatzer M, Hackl H, Sartori B, et al. Differential gene expression in Aspergillus fumigatus induced by human platelets in vitro. Int J Med Microbiol. 2015;305(3):327-38. https://doi.org/10.1016/j.ijmm.2015.01.002.

95. Irmer H, Tarazona S, Sasse C, Olbermann P, Loeffler J, Krappmann S, et al. RNAseq analysis of Aspergillus fumigatus in blood reveals a just wait and see resting stage behavior. BMC Genomics. 2015;16(1):640. https://doi.org/10.1186/s12864-015-1853-1.

96. Oosthuizen JL, Gomez P, Ruan J, Hackett TL, Moore MM, Knight DA, et al. Dual organism transcriptomics of airway epithelial cells interacting with conidia of Aspergillus fumigatus. PLoS One. 2011;6(5):e20527. https://doi.org/10.1371/journal.pone.0020527.

97. McDonagh A, Fedorova ND, Crabtree J, Yu Y, Kim S, Chen D, et al. Sub-telomere directed gene expression during initiation of invasive aspergillosis. PLoS Pathog. 2008;4(9):e1000154. https:// doi.org/10.1371/journal.ppat.1000154.

98.• Ibrahim-Granet O, Dubourdeau M, Latgé J-P, Ave P, Huerre M, Brakhage AA, et al. Methylcitrate synthase from Aspergillus fumigatus is essential for manifestation of invasive aspergillosis. Cell Microbiol. 2008;10(1):134 48. https://doi.org/10.1111/j.14625822.2007.01025. $x$ This report describes the critical role of the A. fumigatus methylcitrate cycle during pulmonary aspergillosis. Via this cycle host-derived amino acids can be integrated in the fungal energy metabolism. This study provides thereby solid proof that amino acids are a main energy source for A. fumigatus during infection of the lung.

99. Fernandes TR, Segorbe D, Prusky D, Di Pietro A. How alkalinization drives fungal pathogenicity. PLoS Pathog. 2017;13(11): e1006621. https://doi.org/10.1371/journal.ppat.1006621.

100. Fu MS, Coelho C, De Leon-Rodriguez CM, Rossi DCP, Camacho E, Jung EH, et al. Cryptococcus neoformans urease affects the outcome of intracellular pathogenesis by modulating phagolysosomal pH. PLoS Pathog. 2018;14(6):e1007144. https://doi.org/10.1371/journal.ppat.1007144 Description of the C. neoformans as a modulator of phagosomal $\mathrm{pH}$ in macrophages. Urease activity enhances the frequency of nonlytic fungal escape from macrophages.

101. Navarathna DHMLP, Lionakis MS, Lizak MJ, Munasinghe J, Nickerson KW, Roberts DD. Urea amidolyase $(D U R 1,2)$ contributes to virulence and kidney pathogenesis of Candida albicans.
PLoS One. 2012;7(10):e48475. https://doi.org/10.1371/journal. pone. 0048475 .

102. Danhof HA, Lorenz MC. The Candida albicans ATO gene family promotes neutralization of the macrophage phagolysosome. Infect Immun. 2015;83(11):4416-26. https://doi.org/10.1128/IAI.00984-15.

103. Lorenz MC, Bender JA, Fink GR. Transcriptional response of Candida albicans upon internalization by macrophages. Eukaryot Cell. 2004;3(5):1076-87. https://doi.org/10.1128/EC.3. 5.1076-1087.2004.

104. Ghosh S, Navarathna DHMLP, Roberts DD, Cooper JT, Atkin $\mathrm{AL}$, Petro TM, et al. Arginine-induced germ tube formation in Candida albicans is essential for escape from murine macrophage line RAW 264.7. Infect Immun. 2009;77(4):1596-605. https://doi. org/10.1128/IAI.01452-08.

105. Westman J, Moran G, Mogavero S, Hube B, Grinstein S. Candida albicans hyphal expansion causes phagosomal membrane damage and luminal alkalinization. MBio. 2018;9(5):e01226-18. https:// doi.org/10.1128/mBio.01226-18 This study provides another explanation for phagosomal alkalinization, where it is described as a consequence and not the cause of hyphal morphogenesis.

106. Jimenez-Lopez C, Collette JR, Brothers KM, Shepardson KM, Cramer RA, Wheeler RT, et al. Candida albicans induces arginine biosynthetic genes in response to host-derived reactive oxygen species. Eukaryot Cell. 2013;12(1):91-100. https://doi.org/10. 1128/EC.00290-12.

107. Kaltdorf M, Srivastava M, Gupta SK, Liang C, Binder J, Dietl A$\mathrm{M}$, et al. Systematic identification of anti-fungal drug targets by a metabolic network approach. Front Mol Biosci. 2016;3:22. https:// doi.org/10.3389/fmolb.2016.00022.

108. Jastrzębowska K, Gabriel I. Inhibitors of amino acids biosynthesis as antifungal agents. Amino Acids. 2015;47(2):227-49. https:// doi.org/10.1007/s00726-014-1873-1.

109. McCarthy MW, Walsh TJ. Amino acid metabolism and transport mechanisms as potential antifungal targets. Int J Mol Sci. 2018;19(3):E909. https://doi.org/10.3390/ijms19030909.

110. Kingsbury JM, McCusker JH. Cytocidal amino acid starvation of Saccharomyces cerevisiae and Candida albicans acetolactate synthase (ilv2 $\Delta$ ) mutants is influenced by the carbon source and rapamycin. Microbiology. 2010;156(Pt 3):929-39. https://doi. org/10.1099/mic.0.034348-0.

111. Kingsbury JM, McCusker JH. Fungal homoserine kinase (thr1 $\Delta$ ) mutants are attenuated in virulence and die rapidly upon threonine starvation and serum incubation. Eukaryot Cell. 2010;9(5):72937. https://doi.org/10.1128/EC.00045-10.

Publisher's Note Springer Nature remains neutral with regard to jurisdictional claims in published maps and institutional affiliations. 